Association for Information Systems AIS Electronic Library (AISeL)

PACIS 2005 Proceedings

Pacific Asia Conference on Information Systems

(PACIS)

December 2005

\title{
Common Structure and Properties of Filtering Systems
}

Junichi Iljima

Tokyo Institute of Technology

Sho Ho

Tokyo Institute of Technology

Follow this and additional works at: http://aisel.aisnet.org/pacis2005

\section{Recommended Citation}

Iljima, Junichi and Ho, Sho, "Common Structure and Properties of Filtering Systems" (2005). PACIS 2005 Proceedings. 91. http://aisel.aisnet.org/pacis2005/91

This material is brought to you by the Pacific Asia Conference on Information Systems (PACIS) at AIS Electronic Library (AISeL). It has been accepted for inclusion in PACIS 2005 Proceedings by an authorized administrator of AIS Electronic Library (AISeL). For more information, please contact elibrary@aisnet.org. 


\title{
Common Structure and Properties of Filtering Systems
}

\author{
Junichi Iijima \\ Graduate School of Decision Science and \\ Technology \\ Tokyo Institute of Technology \\ iijima@me.titech.ac.jp
}

\author{
Sho Ho \\ Graduate School of Decision Science and \\ Technology \\ Tokyo Institute of Technology \\ s-hou@me.titech.ac.jp
}

\begin{abstract}
Recommendation systems have been studied actively since the 1990s. Generally, recommendation systems choose one or more candidates from a set of candidates through a filtering process. Methods of filtering can be divided into two categories: collaborative filtering, in which candidates are chosen based on choices of other persons whose interests or tastes are similar, and content-based filtering, in which items are chosen based on the profile or action history of the recommendee. However, these methods share the same structure in the sense that both of them recommend items based on relevance degrees of items and references, as well as relevance degrees between the recommendee and each reference. Most discussions about recommendation systems focus on the methods of choosing recommended candidates; few focus on foundational concepts of recommendation conditions that systems must satisfy, and problems that current systems have compared with these conditions. In this paper, recommendation systems are reconsidered from the viewpoint of multi-criteria decision making. Conventional filtering methods (e.g., collaborative filtering and content-based filtering) are formulated as linear weighted sum type recommendation systems. Several properties of linear weighted sum type recommendation systems are identified and formulated from the viewpoint of voting.
\end{abstract}

Keywords: Social Choice Theory, Collaborative Filtering, Content-based Filtering, Recommendation System

\section{Introduction}

As an ICT (Information and Communication Technology) application that meets the growing needs of "personalization" in our advanced information society, recommendation systems have been studied actively since the 1990s. ICT-enabled recommendation systems (e.g., online shopping systems that recommend products based on customer profile and history of customer actions; enterprise knowledge portals that send necessary information in a timely manner to each employee according to his/her specialty and position) are infiltrating various aspects of our life. On the other hand, due to the rapid growth of ICT, an enormous amount of information that exceeds the capability of human information processing is now being distributed via various networks. To handle the flood of information, recommendation systems that effectively collect and choose information based on objectives and preferences of users are becoming indispensable.

Early studies of ICT-enabled information recommendation systems include Tapestry (Gold-berg, 1992), GroupLens (Resnik et al., 1994), and Fab (Balabanovic and Shoham, 1997). The term "collaborative filtering" was first used in 1992 by Goldberg et al. in their paper that introduced an information distribution system named Tapestry. In Tapestry, users can set rules such as "if Joe and Bill receive a message, then I would like to receive that message, too" and filter messages based on the rules. The collaborative filtering in Tapestry 
was conducted semi-automatically. In the late 1990s, GroupLens, which conducts collaborative filtering automatically, was developed. GroupLens is a system for collaborative filtering of online news in which the relative degree of users is calculated based on their rating of articles, and articles are recommended based on the rate given by highly relative users. Meanwhile, hybrid recommendation systems that integrate content-based filtering and collaborative filtering have also been developed. Fab, which recommends www pages to users, is a typical hybrid recommendation system. In the Fab system, users receive items that score highly against the user profile, as well as items which are rated highly by a user with a similar profile (Balabanovic and Shoham, 1997).

Generally, recommendation systems choose one or more candidate from a set of candidates through a filtering process. Methods of filtering used in recommendation systems can be divided into two categories. One is collaborative filtering, in which items are chosen based on the choices of other persons whose interests or tastes are similar to those of the recommendee to some degree. Examples of collaborative filtering systems include GroupLens (Resnik et al., 1994), RINGO (Shardanand and Maes, 1995), and Jester (Goldberg et al., 2001). Another method is content-based filtering, in which items are chosen based on the profile or action history of the recommendee. Examples of content-based filtering system include the movie selection system proposed by Alspector et al. (Alspector et al., 1998), MetaSEEk (Beigi et al., 1998), and the book recommend system developed by Mooney and Roy (Mooney and Roy, 2000). These two filtering methods are based on different ideas. However, they share the same structure in the sense that both of them recommend items based on relevance degrees of items and references, as well as relevance degrees between the recommendee and each reference. Highly relevant candidates with highly relevant references are recommended to use filtering systems in both types.

A considerable amount of research has been conducted on recommendation systems, most of which propose concrete designs for software. Meanwhile, few studies have been made about relevant foundational concepts and the theories. Nevertheless, it is important to identify basic conditions that a system needs to meet to be a "recommendation" system. Also it is necessary to check the various proposed recommendation systems to see if they meet these conditions. When we choose from among several candidates based on multiple orders of preference, our action can be understand in a framework similar to the mechanism of voting. The mechanism of voting has been investigated for decades in the field of social choice theory. The correspondence between collaborative filtering and social choice theory has been explored by Pennock et al. (Pennock et al., 2000). Pennock et al. considered collaborative filtering as systems that combine preferences and focused on the concepts of universal domain (UNIV) and unanimity (UNAM) in social choice theory. Base on these concepts, several properties of collaborative filtering systems was identified (Pennock et al., 2000). However, some other important properties of recommendation systems which are related to the voting schemes in social choice theory (e.g., Strategic Manipulability) were not addressed in Pennock et al.'s work.

In this paper, we introduce a new formulation of collaborative filtering and content-based filtering and put our focus on linear weighted sum type recommendation systems. Based on social choice theory, several properties of linear weighted sum type recommendation systems are discussed.

\section{Common Structure of Filtering Systems}


Recommendation systems assume the preference of the recommendee in some way and choose one or more items from a set of items through a filtering process. Collaborative filtering systems choose items based on choices of other persons whose interests or tastes are similar to those of the recommendee to some degree. Content-based filtering systems choose items based on the profile or action history of the recommendee. Recommendation systems choose a set of recommended candidates out of a set of candidates based on a set of references (i.e., other users or various attributes in user profiles), the relevance degree between each reference and the recommendee, and the relevance degree between each reference and candidates.

The concept of recommendation problem is defined as follows:

\section{Definition 1 Recommendation Problem}

A recommendation problem $R P$ is a quadraple $<C, R, \rho, \sigma>$ where:

- $C$ is the set of candidates $C=\left\{c_{j} \mid j=1, . ., m\right\}$,

- $\quad R$ is the set of references $R=\left\{r_{i} \mid i=1, . ., n\right\}$,

- $\rho: R \rightarrow \Re$ is a function that denotes the relevance degree between reference and the recommendee. Here we assume that $\rho(r) \geq 0$ for any $r \in R$, which means that no reference has a negative correlation with the recommendee.

- $\sigma: \mathrm{C} \times \mathrm{R} \rightarrow \mathfrak{R}$ is a function that denotes the relevance degree between candidate and reference. Here we assume that $\sigma(x, r) \geq 0$ for any $x \in C, r \in R$, which means that no reference has a negative correlation with any candidate.

Let us denote the set of recommendation problems by $\mathbf{R P}$ and let

$$
\mathbf{C}=\left\{\mathrm{C}_{0} \mid(\exists<\mathrm{C}, \mathrm{R}, \rho, \sigma>\in \mathbf{R P})\left(\mathrm{C}_{0} \subset \mathrm{C}\right)\right\} .
$$

In a recommendation problem, $\sigma(x, r)$ denotes the relevance degree of reference $r$, and candidate $\mathrm{x}$. For example, in collaborative filtering, $\sigma(\mathrm{x}, \mathrm{r})$ shows the rating that user $\mathrm{r}$ gives to item $\mathrm{x}$, while in content-based filtering, $\sigma(\mathrm{x}, \mathrm{r})$ denotes the value of feature $\mathrm{r}$ of item $\mathrm{x}$. In the case of Grouplens (Resnik et al., 1994), $\rho(\mathrm{r})$ is determined by the correlation between preference of the target user and users who serve as references, thus $\rho(r)$ may have a negative value, while another collaborative filtering system SPARS-J (Ichii et al., 2004) uses the square of correlation to ensure a non-negative $\rho(r)$, and to give a larger weight to highly correlated references. As for the relevance degree between references and candidates $\sigma$, GroupLens (Resnik et al., 1994) uses the difference between the rating a user gave to an item and the average rating of the item, while SPARS-J (Ichii et al., 2004) uses a Boolean variable which represents the user recommended the item or not.

A recommendation system can be seen as a mechanism that gives a subset of candidates for each recommendation problem, which can be formulated as follows:

Definition 2 Recommendation System

A recommendation system is a function $\Phi: \mathbf{R P} \rightarrow \mathbf{C}$ satisfying $\Phi(\mathrm{RP}) \subset \mathrm{C}$ for $\mathrm{RP}=<\mathrm{C}, \mathrm{R}, \rho, \sigma>$ in $\mathbf{R P}$.

Both collaborative filtering and content-based filtering can be seen as a mechanism that recommends items based on the weighted sum of relevance degrees of those items and references, where the weight is determined by the relevance degree between the recommendee and each reference. Here we define this kind of recommendation systems as linear weighted sum type recommendation system. 
Definition 3 Linear Weighted Sum Type

A recommendation system $\Phi: \mathbf{R P} \rightarrow \mathbf{C}$ is linear weighted sum type if and only if for any $\mathrm{RP}=<\mathrm{C}, \mathrm{R}, \rho, \sigma>\in \mathbf{R P}$,

$$
\mathrm{x} \in \Phi(\mathrm{RP}) \Leftrightarrow(\forall \mathrm{y} \in \mathrm{C})(\Sigma(\rho(\mathrm{r}) \sigma(\mathrm{y}, \mathrm{r}) \mid \mathrm{r} \in \mathrm{R}) \leq \Sigma(\rho(\mathrm{r}) \sigma(\mathrm{x}, \mathrm{r}) \mid \mathrm{r} \in \mathrm{R}))
$$

In other words, a linear weighted sum type recommendation system recommends the candidate that has the largest weighted sum of $\sigma(\mathrm{x}, \mathrm{r})$, where $\rho(\mathrm{r})$, the relevance degrees between the reference $r$ and the recommendee, is used as the weight of $\sigma(x, r)$.

Let us show an example of a collaborative filtering system in order to explain the mechanism of linear weighted sum type recommendation system in detail.

Example 1 Consider the recommendation system of a video rental store ${ }^{1}$. Let the set of references (users who have rated movies in the store) be $\mathrm{R}=\{\mathrm{Mr}$. Soga, Mr. Okuno, Mr. Miura $\}$ Let the set of candidates (titles of movies in the store) be

$\mathrm{C}=\{$ Good Bye Lenin, The Chirusoku Summer, Kill Bill Vol.2, Roswell, Ja Zuster Nee Zuster, Jeepers Creepers II \}.

Let the correlation of references and the recommendee be $\rho($ Mr. Soga $)=0.5, \quad \rho($ Mr. Okuno $)=0.6, \quad \rho($ Mr. Miura $)=0.4$

Suppose that the relevance degree between each reference and item are given in Table 1.

Table 1: the relevance degree between each reference and item

\begin{tabular}{|c|c|c|c|c|c|c|c|}
\hline$\sigma(\mathrm{x}, \mathrm{r})$ & $\begin{array}{c}\text { Good } \\
\text { Bye } \\
\text { Lenin }\end{array}$ & $\begin{array}{c}\text { The } \\
\text { Chirusoku } \\
\text { Summer }\end{array}$ & $\begin{array}{c}\text { Kill } \\
\text { Bill } \\
\text { Vol.2 }\end{array}$ & Roswell & $\begin{array}{c}\text { Ja } \\
\text { Zuster } \\
\text { Nee } \\
\text { Zuster }\end{array}$ & $\begin{array}{c}\text { Jeepers } \\
\text { Creepers } \\
\text { II }\end{array}$ & $\rho(\mathrm{r})$ \\
\hline Mr. Soga1 & 1 & 2 & 0 & 5 & 4 & 3 & 0.5 \\
\hline Mr. Okuno2 & 2 & 4 & 1 & 3 & 5 & 0 & 0.6 \\
\hline Mr. Miura3 & 3 & 4 & 0 & 5 & 1 & 3 & 0.4 \\
\hline$\Sigma(\rho(\mathrm{r}) \sigma(\mathrm{x}, \mathrm{r}))$ & 2.9 & 5 & 0.6 & 6.3 & 5.4 & 2.7 & \\
\hline
\end{tabular}

The bottom row of Table 1 shows weighted sums of relevance degrees between references and candidates. In this case, "Roswell" has the largest weighted sum

$$
6.3=5 \times 0.5+3 \times 0.6+5 \times 0.4 \text {. }
$$

Thus, $\Phi(\mathrm{RP})=\{$ “Roswell” $\}$. "Roswell" will be recommended by the system.

A recommendation system determines a set of recommended candidates $\Phi(R P)$ for each recommendation problem $\mathrm{RP}=<\mathrm{C}, \mathrm{R}, \rho, \sigma>$. In many cases, the set of recommended candidates $\Phi(\mathrm{RP})$ is determined by a weak order on C. A weak order is a binary relation that satisfies completeness, transitivity and reflexivity (Kelly, 1998). Hereafter, we denote the set of weak orders on $\mathrm{C}$ by $\operatorname{Ord}=\{\Theta \subset \mathrm{C} \times \mathrm{C} \mid \Theta$ is a weak order on $\mathrm{C}\}$.

\section{Definition 4 Recommendation Order}

A recommendation order induced by $\boldsymbol{\Psi}$ is an ordering on $\mathrm{C}$ determined by a function $\Psi: \mathbf{R P} \rightarrow$ Ord satisfying $\Psi(\mathrm{RP}) \subset \mathrm{C} \times \mathrm{C}$ for $\mathrm{RP}=<\mathrm{C}, \mathrm{R}, \rho, \sigma>$.

\footnotetext{
${ }^{1}$ Movie titles shown in this example were obtained from TSUTAYA Online (http://www.tsutaya.co.jp/).
} 
To make it easier to understand, hereafter we denote $\Psi(\mathrm{RP})$ by $\preceq_{\Psi(\mathrm{RP})}$.

Definition 5 Recommendation System based on Recommendation Order When a recommendation system $\Phi: \mathbf{R P} \rightarrow \mathbf{C}$ satisfies the condition

$$
\mathrm{x} \in \Phi(\mathrm{RP}) \Leftrightarrow(\forall \mathrm{y} \in \mathrm{C})\left(\mathrm{y} \preceq_{\Psi(\mathrm{RP})} \mathrm{x}\right)
$$

it is called a recommendation system based on recommendation order induced by $\Psi: \mathbf{R P} \rightarrow$ Ord.

In other words, recommended candidates in $\Phi(\mathrm{RP})$ are maximum elements in the weak order on $\mathrm{C}$ induced by $\Psi \preceq_{\Psi(\mathrm{RP})}$.

The following proposition can be derived directly from the above definition.

Proposition $1 \mathrm{~A}$ linear weighted sum type recommendation system is determined by the order induced by $\Psi$ defined by:

$$
\mathrm{x} \preceq_{\Psi(\mathrm{RP})} \mathrm{y} \Leftrightarrow \Sigma(\rho(\mathrm{r}) \sigma(\mathrm{y}, \mathrm{r}) \mid \mathrm{r} \in \mathrm{R}) \leq \Sigma(\rho(\mathrm{r}) \sigma(\mathrm{x}, \mathrm{r}) \mid \mathrm{r} \in \mathrm{R})
$$

\section{Three Properties of Recommendation Systems}

In this chapter, we formulate three properties of recommendation systems. These properties have been discussed in the context of social choice theory (Saeki, 1980) and are especially important in the study of recommendation systems.

The first property is a general postulate for discussions about multi-criteria decision making.

\section{Definition 6 Pareto Optimality}

Let $\Phi$ be a recommendation system. $\Phi$ satisfies the Pareto optimality condition if and only if for any recommendation problem $\mathrm{RP}=\langle\mathrm{C}, \mathrm{R}, \rho, \sigma>$, the following condition holds:

$$
(\forall \mathrm{x} \in \mathrm{C})((\exists \mathrm{y} \in \Phi(\mathrm{RP}))(\forall \mathrm{r} \in \mathrm{R})(\sigma(\mathrm{y}, \mathrm{r}) \leq \sigma(\mathrm{x}, \mathrm{r})) \Rightarrow \mathrm{x} \in \Phi(\mathrm{RP}))
$$

In other words, recommendation system $\Phi$ satisfies the Pareto optimality condition in the case that, for any reference $r$, if any candidate worse than candidate $\mathrm{x}$ (in the sense of $\sigma$ ) is recommended then $\mathrm{x}$ will be recommended by $\Phi$ as well.

The next property is about the possibility of a reference $r$ to affect the results of the recommendation by making a fraud about the value of $\sigma$.

\section{Definition 7 Strategic Manipulability}

Recommendation system $\Phi$ satisfies Strategic Manipulability condition if there exist a recommendation problem $\mathrm{RP}=<\mathrm{C}, \mathrm{R}, \rho, \sigma>$, a candidate $\hat{x} \in \mathrm{C}$, and a reference $\mathrm{r}_{0} \in \mathrm{R}$, where $\hat{x}$ is the most highly relevant candidate to $\mathrm{r}_{0}$, in case that $\hat{x}$ is not recommended, $\mathrm{r}_{0}$ can manipulate the result of recommendation to make $\hat{x}$ recommended by changing relevance degrees of several candidates. That is,

$$
\text { if }(\forall \mathrm{y} \in \mathrm{C})\left(\hat{x} \neq \mathrm{y} \Rightarrow \sigma\left(\mathrm{y}, \mathrm{r}_{0}\right)<\sigma\left(\hat{x}, \mathrm{r}_{0}\right)\right) \wedge \hat{x} \notin \Phi(\mathrm{RP})
$$

then a $\sigma$ satisfying the following condition can be defined:

$$
\sigma\left|\mathrm{C} \times\left(\mathrm{R}-\left\{\mathrm{r}_{0}\right\}\right)=\sigma^{\prime}\right| \mathrm{C} \times\left(\mathrm{R}-\left\{\mathrm{r}_{0}\right\}\right) \quad \text { and } \quad \hat{x} \in \Phi\left(\mathrm{RP}^{\prime}\right) \quad \text { where } \mathrm{RP}=<\mathrm{C}, \mathrm{R}, \rho, \sigma^{\prime}>
$$

When the solution $\Phi(\mathrm{RP})$ given by a recommendation system $\Phi$ has already been recommended, it is natural to recommend the first runner-up in the set of candidates. The 
sufficient condition for this is that the recommendation order on the set of candidates $\mathrm{C}$ coincides with the one on $C-\Phi(R P)$.

This property is defined as follows:

\section{Definition 8 Independence of Solutions}

Let $\Phi$ be a recommendation system based on a recommendation order induced by $\Psi$. $\Phi$ satisfies strong independence of solutions condition if for any recommendation problem $\mathrm{RP}=<\mathrm{C}, \mathrm{R}, \rho, \sigma>$ and $\mathrm{RP}^{\prime}=<\mathrm{C}-\Phi(\mathrm{RP}), \mathrm{R}, \rho, \sigma^{\prime}>$,

$$
\begin{gathered}
\text { when }(\forall \mathrm{r} \in \mathrm{R})(\quad \forall \mathrm{x}, \mathrm{y} \in \mathrm{C}-\Phi(\mathrm{RP}))\left(\sigma^{\prime}(\mathrm{x}, \mathrm{r}) \leq \sigma^{\prime}(\mathrm{y}, \mathrm{r}) \Rightarrow \sigma(\mathrm{x}, \mathrm{r}) \leq \sigma(\mathrm{y}, \mathrm{r})\right), \\
\preceq_{\Psi(\mathrm{RP})} \mid(\mathrm{C}-\Phi(\mathrm{RP})) \times(\mathrm{C}-\Phi(\mathrm{RP}))=\preceq_{\Psi(\mathrm{RP})}
\end{gathered}
$$

$\Phi$ satisfies weak independence of solutions condition if for any recommendation problem $\mathrm{RP}=<\mathrm{C}, \mathrm{R}, \rho, \sigma>\quad$ and $\quad \mathrm{RP}^{\prime}=<\mathrm{C}-\Phi(\mathrm{RP}), \mathrm{R}, \rho, \sigma \mid(\mathrm{C}-\Phi(\mathrm{RP})) \times \mathrm{R}>$,

$$
\preceq_{\Psi(\mathrm{RP})} \mid(\mathrm{C}-\Phi(\mathrm{RP})) \times(\mathrm{C}-\Phi(\mathrm{RP}))=\preceq_{\Psi(\mathrm{RP})}
$$

The following lemma is derived from the definition above. (All proofs of lemmas and theorems are shown in the appendix.)

Lemma 1 Let $\Phi$ be a recommendation system based on an order induced by $\Psi$, $\Phi$ satisfies weak independence of solutions condition if $\Phi$ satisfies strong independence of solutions condition.

\section{Properties of Linear Weighted Sum Type Recommendation Systems}

In this chapter, we discuss the relationship between linear weighted sum type recommendation systems and the three properties defined in the last chapter. First, with respect to Pareto optimality, linear weighted sum type recommendation systems have the following properties.

Theorem 1 Linear weighted sum recommendation systems satisfy the Pareto optimality condition.

As the next example shows, strategic manipulation is possible in linear weighted sum type recommendation systems.

Example 2 Let $\mathrm{C}=\{\mathrm{x}, \mathrm{y}, \mathrm{z}\}, \mathrm{R}=\left\{\mathrm{r}_{1}, \mathrm{r}_{2}, \mathrm{r}_{3}\right\}$, and

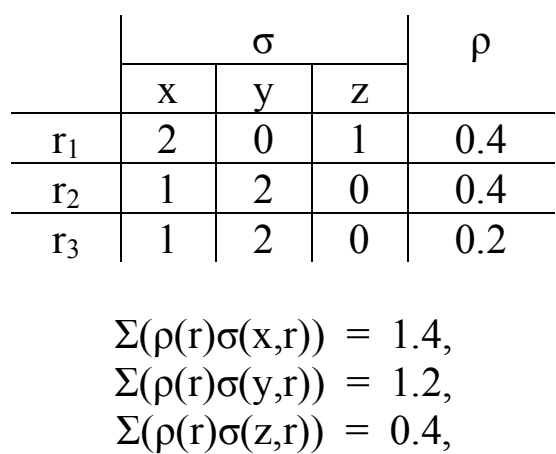


Thus, $\Phi(R P)=\{x\}$. The choice of $r_{2}$ is not recommended. Now assume that $r_{2}$ changes the rating of candidates (as shown below), while ratings made by other references are not changed.

\begin{tabular}{|c|c|c|c|c|}
\hline & \multicolumn{3}{|c|}{$\sigma$} & \multirow[t]{2}{*}{$\rho$} \\
\hline & $\mathrm{X}$ & $\mathrm{y}$ & $\mathrm{Z}$ & \\
\hline $\mathrm{r}_{1}$ & 2 & 0 & 1 & 0.4 \\
\hline$r_{2}$ & 0 & 2 & 1 & 0.4 \\
\hline$r_{3}$ & 1 & 2 & 0 & 0.2 \\
\hline & $\Sigma(\rho)$ & $\begin{array}{l}5(y, \\
(z,\end{array}$ & $\begin{array}{l}= \\
=\end{array}$ & \\
\hline
\end{tabular}

Thus, $\Phi(\mathrm{RP})=\{\mathrm{y}\}$. In this example, $\mathrm{r}_{2}$ succeeded in putting its own choice in the set of recommended candidates by manipulating its own rating of candidates.

Theorem 2 Any linear weighted sum type recommendation system $\Phi$ satisfies the strategic manipulability condition.

The fact that linear weighted sum type recommendation systems satisfy the strategic manipulability condition means that, in collaborative filtering, a user may lead the result of recommendation to his/her intended direction by making a fraud about $\sigma$ for certain candidates. This is one of the problems conventional recommendation systems have.

The next proposition is about the condition of not allowing strategic manipulation.

Proposition 2 For a linear weighted sum type recommendation system $\Phi$, a recommendation problem $\mathrm{RP}=<\mathrm{C}, \mathrm{R}, \rho, \sigma>$, a candidate $\hat{x} \in \mathrm{C}$, and a reference $\mathrm{r}_{0} \in \mathrm{R}$, when $\hat{x}$ has the highest relative degree with $\mathrm{r}_{0}$ and there exists a $\hat{y}$ such that $\hat{x}<_{\Psi(\mathrm{RP})} \hat{y}$,

$$
\hat{x}<_{\Psi(\mathrm{RP})} \hat{y} \text { where } \mathrm{RP}^{\prime}=<\mathrm{C}, \mathrm{R}, \rho, \sigma^{\prime}>
$$

holds if and only if $\sigma\left(\hat{x}, \mathrm{r}_{0}\right)=\sigma^{\prime}\left(\hat{x}, \mathrm{r}_{0}\right)$ and $\mathrm{r}_{0}$ changes $\sigma^{\prime}\left(\hat{y}, \mathrm{r}_{0}\right)$ within the range restricted by the following condition:

$$
\text { (condition) } \rho\left(\mathrm{r}_{0}\right)\left(\sigma\left(\hat{y}, \mathrm{r}_{0}\right)-\sigma^{\prime}\left(\hat{y}, \mathrm{r}_{0}\right)\right)<\Sigma(\rho(\mathrm{r})(\sigma(\hat{y}, \mathrm{r})-\sigma(\hat{x}, \mathrm{r})))
$$

This proposition shows that the change of recommendation order would not occur if the range of change on each $\sigma\left(\hat{y}, \mathrm{r}_{0}\right)$ is restricted by the previous condition.

Let us illustrate this condition with an example.

Example 3 Using the case described in example 2, assume that $r_{2}$ can only change values of $\sigma^{\prime}$ within the range restricted by the condition defined in proposition 2 . Since $\Sigma(\rho(\mathrm{r})(\sigma(\mathrm{x}, \mathrm{r})-\sigma(\mathrm{y}, \mathrm{r})))=1.4-1.2=0.2$, according to the condition,

$$
\frac{\rho\left(\mathrm{r}_{2}\right) \sigma\left(\mathrm{x}, \mathrm{r}_{2}\right)-0.2}{\rho\left(\mathrm{r}_{2}\right)}=\frac{0.4 \times 1-0.2}{0.4}=0.5<\sigma^{\prime}\left(\mathrm{x}, \mathrm{r}_{2}\right) .
$$

Let $\sigma^{\prime}\left(\mathrm{x}, \mathrm{r}_{2}\right)=0.5+\varepsilon$, then

$$
\begin{gathered}
\Sigma(\rho(\mathrm{r}) \sigma(\mathrm{x}, \mathrm{r}))=1.2+0.4 \varepsilon \\
\Sigma(\rho(\mathrm{r}) \sigma(\mathrm{y}, \mathrm{r}))=1.2, \\
\Sigma(\rho(\mathrm{r}) \sigma(\mathrm{z}, \mathrm{r}))=0.8,
\end{gathered}
$$


The recommendation order does not change.

As shown below, linear weighted sum type recommendation systems satisfy the weak independence of solutions condition but do not satisfy the strong independence of solutions condition. That is to say that, when all candidates in solution $\Phi(R P)$ given by a recommendation system $\Phi$ have already been recommended and it is necessary to choose from other candidates, if the relative degree $\sigma(\mathrm{x}, \mathrm{r})$ of some $\mathrm{x}$ and $\mathrm{r}$ is changed, we could not simply recommend the first runner-up, even in case that the order of $\sigma(\mathrm{x}, \mathrm{r})$ did not change.

The following example shows that in general a linear weighted sum type recommendation system does not satisfy the strong independence of solutions condition.

Example 4 Let $\mathrm{C}=\{\mathrm{x}, \mathrm{y}, \mathrm{z}\}, \mathrm{R}=\left\{\mathrm{r}_{1}, \mathrm{r}_{2}, \mathrm{r}_{3}\right\}$,

Thus,

\begin{tabular}{c|c|c|c|c}
\multirow{2}{*}{} & \multicolumn{3}{|c|}{$\sigma$} & $\rho$ \\
\cline { 2 - 4 } & $\mathrm{x}$ & $\mathrm{y}$ & $\mathrm{z}$ & \\
\hline $\mathrm{r}_{1}$ & 2 & 0 & 1 & 0.4 \\
\hline $\mathrm{r}_{2}$ & 1 & 2 & 0 & 0.2 \\
\hline $\mathrm{r}_{3}$ & 0 & 1 & 2 & 0.4
\end{tabular}

$$
\begin{aligned}
& \Sigma(\rho(\mathrm{r}) \sigma(\mathrm{x}, \mathrm{r}))=1.2 \\
& \Sigma(\rho(\mathrm{r}) \sigma(\mathrm{y}, \mathrm{r}))=1.0 \\
& \Sigma(\rho(\mathrm{r}) \sigma(\mathrm{z}, \mathrm{r}))=0.8
\end{aligned}
$$

$\mathrm{z}<_{\Psi(\mathrm{RP})} \mathrm{y}<_{\Psi(\mathrm{RP})} \mathrm{x}$ and $\Phi(\mathrm{RP})=\{\mathrm{x}\} . \quad$ Now let $\mathrm{C}^{\prime}=\mathrm{C}-\Phi(\mathrm{RP})=\{\mathrm{y}, \mathrm{z}\}$ and define $\sigma^{\prime}$ as follows:

\begin{tabular}{c|c|c}
\multirow{2}{*}{} & \multicolumn{2}{|c}{$\sigma^{\prime}$} \\
\cline { 2 - 3 } & $\mathrm{y}$ & $\mathrm{z}$ \\
\hline $\mathrm{r}_{1}$ & 0 & 1 \\
\hline $\mathrm{r}_{2}$ & 1 & 0 \\
\hline $\mathrm{r}_{3}$ & 0 & 1
\end{tabular}

The condition $(\forall \mathrm{r} \in \mathrm{R})\left(\forall \mathrm{x}, \mathrm{y} \in \mathrm{C}^{\prime}\right)\left(\sigma^{\prime}(\mathrm{x}, \mathrm{r}) \leq \sigma^{\prime}(\mathrm{y}, \mathrm{r}) \Leftrightarrow \sigma(\mathrm{x}, \mathrm{r}) \leq \sigma(\mathrm{y}, \mathrm{r})\right)$ holds. However, $\mathrm{y}<\Psi(\mathrm{RP}) \mathrm{z}$ since

$$
\begin{aligned}
& \Sigma\left(\rho(\mathrm{r}) \sigma^{\prime}(\mathrm{y}, \mathrm{r})\right)=0.4, \\
& \Sigma\left(\rho(\mathrm{r}) \sigma^{\prime}(\mathrm{z}, \mathrm{r})\right)=0.8,
\end{aligned}
$$

Therefore, $\Phi$ does not satisfy the strong independence of solutions condition.

On the other hand, in case that $\sigma^{\prime}$ is a mapping similar to $\sigma$ except for that $\mathrm{x}$ is excluded from the domain of $\sigma^{\prime}$, it is easy to presume that the result would be $\mathrm{z}<_{\Psi\left(\mathrm{RP} \mathrm{P}^{\prime}\right)}$ y since the weighted sum of the relevance degrees does not change. In this case, recommending the first runner-up would be meaningful. The next theorem shows that $\Phi$ satisfies the weak independence of solutions condition.

Theorem 3 Linear weighted sum recommendation systems satisfy the weak independence of solutions condition, but do not satisfy the strong independence of solutions condition.

\section{Conclusion}


Most of the preceding discussion about recommendation systems focuses on the method of choosing recommended candidates, which is a relatively practical matter compared with the basic concept of recommendation. Few discussions have been made on the basic concept of recommendation, the conditions that recommendation systems must satisfy, and the problems that current recommendation systems have in relation to these conditions.

In this paper, recommendation systems are reconsidered from the viewpoint of multi-criteria decision making. Conventional filtering methods (e.g., collaborative filtering and content-base filtering) are formulated as linear weighted sum type recommendation systems. Also, several properties of linear weighted sum type recommendation systems are identified and formulated from the viewpoint of voting.

\section{References}

Alspector J., Kolcz A., and Karunanithi N. "Comparing feature-based and clique-based user models for movie selection," Proceedings of the Third ACM Conference on Digital Libraries, Pittsburgh, PA, USA, Ian Witten (eds.), ACM Press, New York, USA, 1998, pp. 11-18.

Balabanovic, M., and Shoham, Y. "Content-Based, Collaborative Recommendation," Communications of the ACM (Association for Computing Machinery), (40:3), 1997, pp. 66-72.

Beigi M., Benitez A.B., and Chang S. "MetaSEEk: A Content-Based Meta-Search Engine for Images," Proceedings of the SPIE 1998 Conference on Storage and Retrieval for Image and Video Databases, San Jose, CA, USA, SPIE, Bellingham, WA, USA, 1998, pp. 118-128.

Goldberg, D.,Nichols, D., Oki, B., and Terry, D. "Using Collaborative Filtering to Weave an Information Tapestry," Communications of the ACM (Association for Computing Machinery), (35:12), 1992, pp. 61-69.

Goldberg K., Roeder T., Gupta D., and Perkins C. "Eigentaste: A constant time collaborative filtering algorithm," Information Retrieval, (4:2), 1999, pp 133-151.

Ichii, M., Yamamoto, T., Yokomori, R., and Inoue, K. "Collaborative Filtering Method for Software Component Recommendation," Technical Report of IEICE (the Institute of Electronics, Information and Communication Engineers), (104:243), 2004, pp. 7-12.

Kelly, J. S. Social Choice Theory: An Introduction, Springer-Verlag, Berlin, 1988.

Mooney R. and Roy L. "Content-based book recommending using learning for text categorization," Proceedings of the Fifth ACM Conference on Digital Libraries, San Antonio, Texas, USA, ACM Press, New York, USA, 2000, pp. 195-204.

Pennock D. M.,Horvitz, E., and Giles, C. L. "Social Choice Theory and Recommender Systems: Analysis of the Axiomatic Foundations of Collaborative Filtering," Proceedings of the Seventeenth National Conference on Artificial Intelligence, Twelfth Conference on Innovative Applications of Artificial Intelligence, Austin, Texas, USA, AAAI Press, Menlo Park, California USA, 2000, pp. 729-734.

Resnik, P.,Iacovou N., Suchak, M., Bergstrom P., Riedl J. "GroupLens: An Open Architecture for Collaborative Filtering of Netnews," Proceedings of the 1994 Computer Supported Cooperative Work Conference, Chapel Hill, North Carolina, USA, J. B. Smith, F. D. Smith and T. W. Malone (eds.), ACM Press, New York, USA, 1994, pp. 175-186.

Saeki Y. Kimekata No Ronri: Syakaiteki Kettei Riron Heno Syoutai, Tokyo Daigaku Syuppankai, Tokyo, 1980

Shardanand U., and Maes P. "Social information filtering algorithms for automating 'word of mouth'," Proceedings of CHI'95 Conference on Human Factors in Computing Systems, 
Denver, Colorado, USA, Irvin Katz et al. (eds.), ACM Press, New York, USA, 1995, pp. 210-217.

\section{Appendix \\ (Proof of Proposition 1)}

Omitted

Q.E.D.

\section{(Proof of Lemma 1)}

Omitted

Q.E.D.

\section{(Proof of Theorem 1)}

Assume that for any $x \in C$, there exist a $y \in \Phi(R P)$ satisfying $(\forall r \in R)(\sigma(y, r) \leq \sigma(x, r))$.

For any $r \in R, \rho(r)$ is non-negative, therefore, $\rho(r) \sigma(y, r) \leq \rho(r) \sigma(x, r)$ for any $r \in R$. Consequently, $\Sigma(\rho(\mathrm{r}) \sigma(\mathrm{y}, \mathrm{r})) \leq \Sigma(\rho(\mathrm{r}) \sigma(\mathrm{x}, \mathrm{r}))$ holds. Since $\mathrm{y} \in \Phi(\mathrm{RP})$,

$(\forall \mathrm{z} \in \mathrm{C})(\Sigma(\rho(\mathrm{r}) \sigma(\mathrm{z}, \mathrm{r})) \leq \Sigma(\rho(\mathrm{r}) \sigma(\mathrm{y}, \mathrm{r})))$. Consequently, $(\forall \mathrm{z} \in \mathrm{C})(\Sigma(\rho(\mathrm{r}) \sigma(\mathrm{z}, \mathrm{r})) \leq \Sigma(\rho(\mathrm{r}) \sigma(\mathrm{x}, \mathrm{r})))$. Thus $\mathrm{x} \in \Phi(\mathrm{RP})$. $\Phi$ satisfies the Pareto optimality condition.

Q.E.D.

\section{(Proof of Theorem 2)}

The proof is obvious from example 2 .

Q.E.D.

\section{(Proof of Proposition 2)}

To prove that $\hat{x}<_{\Psi(\mathrm{RP})} \hat{y}$, it is necessary and sufficient to prove $\Sigma\left(\rho(\mathrm{r}) \sigma^{\prime}(\hat{x}, \mathrm{r})\right)<\Sigma\left(\rho(\mathrm{r}) \sigma^{\prime}(\hat{y}, \mathrm{r})\right)$. From the assumption, for any $\mathrm{r}$ except $\mathrm{r}_{0}$, value of $\sigma(\hat{x}, \mathrm{r})$ and $\sigma^{\prime}(\hat{x}, \mathrm{r})$ are equal.

Since $\sigma\left(\hat{x}, \mathrm{r}_{0}\right)=\sigma^{\prime}\left(\hat{x}, \mathrm{r}_{0}\right)$, the left side of the equation is equal to $\Sigma(\rho(\mathrm{r}) \sigma(\hat{x}, \mathrm{r}))$.

Meanwhile, from the assumption, for any $\mathrm{r}$ except $\mathrm{r}_{0}, \sigma(\hat{y}, \mathrm{r})$ and $\sigma^{\prime}(\hat{y}, \mathrm{r})$ are equal; therefore, the right side of the equation is equal to $\Sigma(\rho(\mathrm{r}) \sigma(\hat{y}, \mathrm{r}))-\rho\left(\mathrm{r}_{0}\right) \sigma\left(\hat{y}, \mathrm{r}_{0}\right)+\rho\left(\mathrm{r}_{0}\right) \sigma^{\prime}\left(\hat{y}, \mathrm{r}_{0}\right)$.

Thus, it is necessary and sufficient to prove that

$$
\Sigma(\rho(\mathrm{r}) \sigma(\hat{x}, \mathrm{r}))<\Sigma(\rho(\mathrm{r}) \sigma(\hat{y}, \mathrm{r}))-\rho\left(\mathrm{r}_{0}\right) \sigma\left(\hat{y}, \mathrm{r}_{0}\right)+\rho\left(\mathrm{r}_{0}\right) \sigma^{\prime}\left(\hat{y}, \mathrm{r}_{0}\right)
$$

which can be transformed to

$$
\rho\left(\mathrm{r}_{0}\right)\left(\sigma\left(\hat{y}, \mathrm{r}_{0}\right)-\sigma^{\prime}\left(\hat{y}, \mathrm{r}_{0}\right)\right)<\Sigma(\rho(\mathrm{r})(\sigma(\hat{y}, \mathrm{r})-\sigma(\hat{x}, \mathrm{r})))
$$

Q.E.D.

\section{(Proof of Theorem 3)}

It is clear from example 4 that in general, linear weighted sum type recommendation systems do not satisfy the strong independence of solutions condition. On the other hand, for any recommendation problem $\mathrm{RP}=<\mathrm{C}, \mathrm{R}, \rho, \sigma>$ and $\mathrm{RP}^{\prime}=<\mathrm{C}-\Phi(\mathrm{RP}), \mathrm{R}, \rho, \sigma \mid(\mathrm{C}-\Phi(\mathrm{RP})) \times \mathrm{R}>$, it is clear that for any $\mathrm{x}, \mathrm{y} \in \mathrm{C}-\Phi(\mathrm{RP}), \Sigma(\rho(\mathrm{r}) \sigma(\mathrm{x}, \mathrm{r}) \mid(\mathrm{C}-\Phi(\mathrm{RP})) \times \mathrm{R})=\Sigma(\rho(\mathrm{r}) \sigma(\mathrm{x}, \mathrm{r}))$ and $\Sigma(\rho(\mathrm{r}) \sigma(\mathrm{y}, \mathrm{r}) \mid(\mathrm{C}-\Phi(\mathrm{RP})) \times \mathrm{R})=\Sigma(\rho(\mathrm{r}) \sigma(\mathrm{y}, \mathrm{r}))$ holds .

If $\mathrm{x} \preceq_{\Psi(\mathrm{RP})} \mathrm{y}$, it is clear from the definition that $\Sigma(\rho(\mathrm{r}) \sigma(\mathrm{x}, \mathrm{r})) \leq \Sigma(\rho(\mathrm{r}) \sigma(\mathrm{y}, \mathrm{r}))$ holds.

Consequently, $\Sigma(\rho(\mathrm{r}) \sigma(\mathrm{x}, \mathrm{r}) \mid(\mathrm{C}-\Phi(\mathrm{RP})) \times \mathrm{R}) \leq \Sigma(\rho(\mathrm{r}) \sigma(\mathrm{y}, \mathrm{r}) \mid(\mathrm{C}-\Phi(\mathrm{RP})) \times \mathrm{R})$ holds, hence, $\mathrm{x} \preceq_{\Psi(\mathrm{RP})} \mathrm{y}$ holds. It is obvious that the opposite also holds. Therefore, linear weighted sum type recommendation systems satisfy the weak independence of solutions condition.

Q.E.D. 